\title{
Stochastic Resonance and Simulation of a Single-mode Laser System with Periodically Modulated Signal
}

\author{
Wang Guowei*, Fu Yan \\ School of General Studies, Nanchang Institute of Science \& Technology, Nanchang, 330108, China \\ *corresponding author email:501284253@qq.com
}

Keywords: noise; simulation; single-mode laser; intensity correlation function; stochastic resonance

\begin{abstract}
The time evolution of intensity correlation function $C(t)$ in a single-mode laser driven by the coloured pump noise with signal modulation and the quantum noise with cross-correlation between the real and imaginary parts is studied by using the linear approximation method. In the case of the pump noise self-correlation time $\tau<<1$, it can be found that: (i)when modulation signal frequency $\Omega$ increases, the time evolution of $C(t)$ experiences a changing process, that is, from the monotonous descending to the descending after the appearance of an initial flat, and finally to the periodically surging with descending envelope; (ii)When the net gain $a_{0}$ increases, it experiences a repeatedly changing process, that is, from the monotonous descending to monotonous rise, and to the appearance of a maximum, finally to monotonous descending again. However, in the case of $\tau \gg>1$, the time evolution of $C(t)$ only exhibits periodically surging with descending envelope.
\end{abstract}

\section{Introduction}

On account of the great influence of noise and input signal on the dynamic behavior of the non-linear stochastic system, the study of dynamic behavior for such system has attracted researchers' wide attention. Since Fulinski et al. first studied the influence of cross-correlation noise on the statistical properties of non-linear system, Cao et al. have made a more detailed study of one dimensional stochastic system driven by cross-correlation noise, and Zhu et.al. have introduced cross-correlation noise into the laser system and studied the stationary state property of the laser system driven by pump noise and quantum noise with cross-correlation between real and imaginary parts. We have studied the dynamic property of a single-mode laser system driven by pump noise and quantum noise with cross-correlation between the real and imaginary parts. However, in the above-mentioned works, scholars laid more emphasis on the influence of cross-correlation noise on the dynamic behavior of the non-linear stochastic system, giving little consideration to the influence of the signal on the dynamic behavior of the laser system. Dykman has introduced into the laser system the modulation of noise by the signal.The noise modulated by the signal can better describe the laser system than the ordinary noise, because when a laser system is modulated by signal, the noise of the system will simultaneously be modulated by the signal. As a result, the modulated noise will highly affect the dynamic behavior in the laser system. The study of the function of the modulation signal in the time evolution of the laser intensity correlation function in a single-mode laser system driven by the modulated noise can shows, in a certain aspect, how the modulation signal affects the dynamic behavior of laser system. Thus it is of great theoretic significance. The basic statistic quantity in describing the dynamic property of a laser system is the intensity correlation function, from which the power spectrum, output power, correlation time and the variance of laser intensity can be educed. With the intensity correlation function we can further study the phenomenon of stochastic resonance (SR), providing a theoretic base for optimizing the laser system. Therefore, our study of is great application value in the field of laser communication.

The time evolution of intensity correlation function for a loss-noise model of a single-model laser system driven by colored pump noise with signal modulation and the quantum noise with 
cross-correlation between the real and imaginary parts is studied adopting the linear approximation method in this paper. We also provide a detailed analysis of the influence of the net gain $a_{0}$, the amplitude $B$ and frequency $\Omega$ of modulation signal on the time evolution of the intensity correlation function. Because of the application of the unified colored noise approximation in the process of solving the Langevin equation, we will discuss the time evolution of the intensity correlation function in the case of the pump noise self-correlation time $\tau \gg>1$ only. Consequently, we find that the signal frequency $\Omega$ has great influence on the process of evolution.

\section{The Intensity Correlation Function}

The Langevin equation of amplitude for a loss-noise model of a single-mode laser is given by

$$
\frac{d r}{d t^{\prime}}=a_{0} r-A r^{3}+\frac{Q}{2 r}\left(1-\left|\lambda_{q}\right|\right)+r p_{R}\left(t^{\prime}\right)+\varepsilon_{r}\left(t^{\prime}\right)
$$

Considered the pump noise is modulated by periodically signal $B \cos \Omega t^{\prime}$, thus the Langevin equation of intensity for a loss-noise model of a single-mode laser with an input signal is given by

$$
\frac{d I}{d t^{\prime}}=2 a_{0} I-2 A I^{2}+Q\left(1-\left|\lambda_{q}\right|\right)+2 I p_{R}\left(t^{\prime}\right) B \cos \Omega t^{\prime}+2 \sqrt{I} \varepsilon_{r}\left(t^{\prime}\right)
$$

where noises $p_{R}\left(t^{\prime}\right)$ and $\varepsilon_{r}\left(t^{\prime}\right)$ are correlated in the following form

$$
\begin{array}{ll}
\left\langle p_{R}\left(t^{\prime}\right)\right\rangle=\left\langle\varepsilon_{r}\left(t^{\prime}\right)\right\rangle=0 & \left\langle p_{R}\left(t^{\prime}\right) p_{R}(s)\right\rangle=\frac{P}{2 \tau} e^{-\frac{\left|s-t^{\prime}\right|}{\tau}} \\
\left\langle\varepsilon_{r}\left(t^{\prime}\right) \varepsilon_{r}(s)\right\rangle=Q\left(1+\left|\lambda_{q}\right|\right) \delta\left(t^{\prime}-s\right) & \left\langle p_{R}\left(t^{\prime}\right) \varepsilon_{r}(s)\right\rangle=0
\end{array}
$$

In equations (1), (2) and (3), $a_{0}$ and $A$ represent the net gain coefficient and self-saturation coefficient, respectively; $I$ is the laser intensity; $B$ is the amplitude of a modulation signal, $\Omega$ is the signal frequency; $p_{R}\left(t^{\prime}\right)$ is the real part of the pump noise, and $\varepsilon_{r}\left(t^{\prime}\right)$ is the quantum noise of phase lock; $Q$ and $P$ are the intensities of the pump noise and the quantum noise, respectively; $\tau$ is the pump noise self-correlation time; $\lambda_{q}$ is the cross-correlation coefficient between the real part and the imaginary part of the quantum noise, and $-1 \leq \lambda_{q} \leq 1$.

Acorrding to nonlinear approximation, let $I=I_{0}+\delta\left(t^{\prime}\right)$, where $I_{0}=\frac{a_{0}}{A}$, is the deterministic steady-state intensity, and $\delta\left(t^{\prime}\right)$ is the perturbational term. We linearize Eq. (2) around the deterministic steady-state intensity $I_{0}$, thus the linear equation of the laser intensity is found to be

$$
\frac{d \delta\left(t^{\prime}\right)}{d t^{\prime}}=-\gamma \delta\left(t^{\prime}\right)+2 I_{0} p_{R}\left(t^{\prime}\right) B \cos \Omega t^{\prime}+2 \sqrt{I_{0}} \varepsilon_{r}\left(t^{\prime}\right)+Q\left(1-\left|\lambda_{q}\right|\right)
$$

where $\gamma=2 a_{0}$.

The solution of Eq.(4) has the following form

$$
\delta\left(t^{\prime}\right)=e^{\gamma^{\prime}}\left[\frac{P\left(1-\left|\lambda_{q}\right|\right)}{\gamma}\left(e^{\gamma^{\prime}}-1\right)+2 I_{0} B \int_{0}^{t^{\prime}} p_{R}(s) \cos \Omega s e^{\gamma s} d s+2 \sqrt{I_{0}} \int_{0}^{t^{\prime}} \varepsilon_{r}(s) e^{\gamma s} d s\right]
$$

According to the steady-state mean intensity correlation function (SSMICF) defined by

$$
C(t)=\lim _{t^{\prime} \rightarrow \infty} \overline{\left\langle I\left(t^{\prime}\right) I\left(t^{\prime}+t\right)\right\rangle}=\lim _{t^{\prime} \rightarrow \infty}\left(\frac{\Omega}{2 \pi} \int_{t^{\prime}}^{t^{\prime}+\frac{2 \pi}{\Omega}}\left\langle I\left(t^{\prime}\right) I\left(t^{\prime}+t\right)\right\rangle d t^{\prime}\right)
$$

the SSMICF is obtained by Solving Eq.(5)

$$
C(t)=\left(I_{0}+\frac{Q\left(1-\left|\lambda_{q}\right|\right)}{\gamma}\right)^{2}+\left(\frac{2 I_{0} Q\left(1+\left|\lambda_{q}\right|\right)}{\gamma}+\frac{I_{0}^{2} B^{2} P\left(2 \pi \gamma^{2}+\Omega^{3}\right)\left(\Omega^{2}-\gamma^{2}+\tau^{-2}\right)}{\tau^{2} \Omega \gamma\left(k_{1}^{2}+\Omega^{2}\right)\left(k_{2}^{2}+\Omega^{2}\right)\left(\gamma^{2}+\Omega^{2}\right)}\right) e^{-\gamma|t|}
$$




$$
+\frac{I_{0}^{2} B^{2} P\left(\Omega^{2}+\gamma^{2}-\tau^{-2}\right)}{\tau\left(k_{1}^{2}+\Omega^{2}\right)\left(k_{2}^{2}+\Omega^{2}\right)} e^{-\frac{|t|}{\tau}} \cos \Omega t+\frac{2 \Omega I_{0}^{2} B^{2} P}{\tau^{2}\left(k_{1}^{2}+\Omega^{2}\right)\left(k_{2}^{2}+\Omega^{2}\right)} e^{-\frac{|t|}{\tau}} \sin \Omega|t|
$$

here, $k_{1}=\gamma-\tau^{-1}, \quad k_{2}=\gamma+\tau^{-1}$, and $\tau \neq \frac{1}{2 a_{0}}$.

From Eq.(6) we can see that there are two characteristics of the intensity correlation function $C(t)$ : firstly, because of pump noise has "colored" character, thus there are two timescales of the $C(t)$; secondly, because of the pump noise is modulated by signal, so that the $C(t)$ is a periodicity function of the signal frequency $\Omega$.

In the following, we will discuss the influence of the net gain $a_{0}$, and the frequency $\Omega$ of modulation signal on the time evolution of the $C(t)$ based on Eq.(6). Furthermore, due to the use of unified colored noise approximation in solving Eq.(1), we will only discuss the time evolution of $C(t)$ in the cases of $\tau>>1$.

\section{The Influence of Frequency $\Omega$ of Modulation Signal on the Time Evolution of the Intensity Correlation Function $C(t)$}
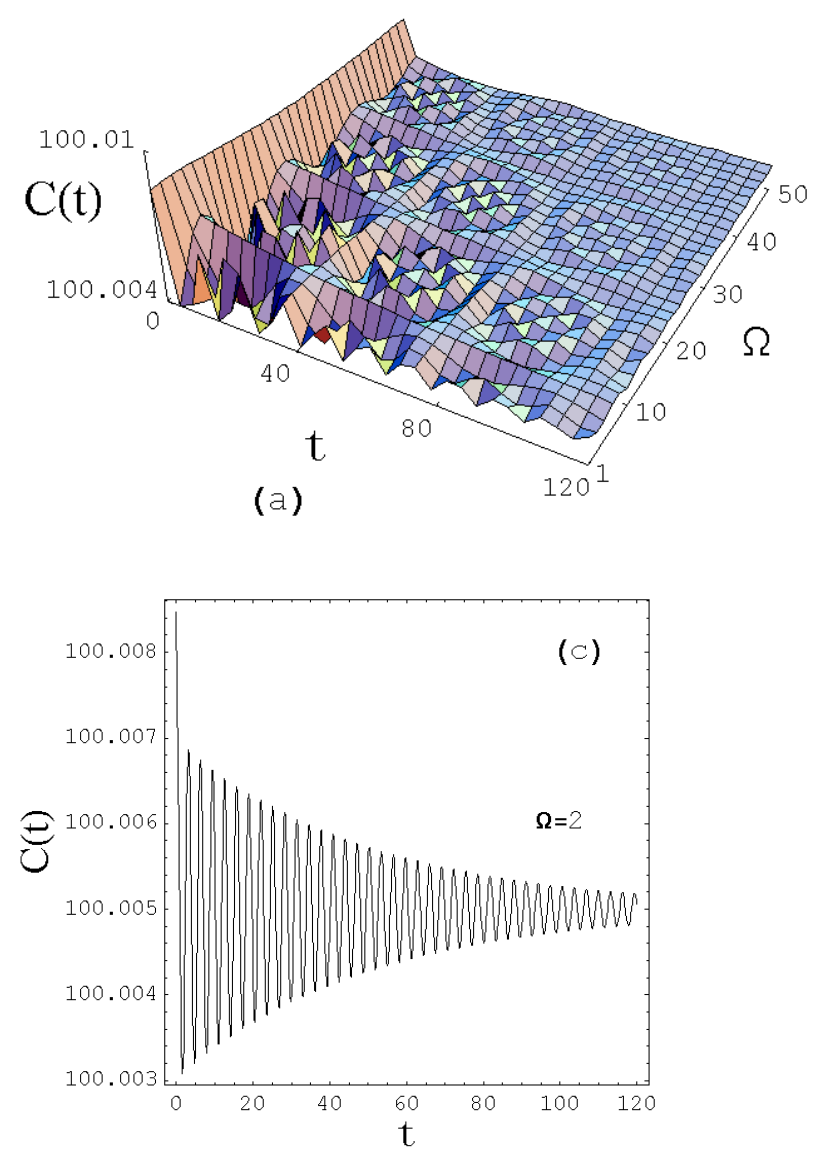
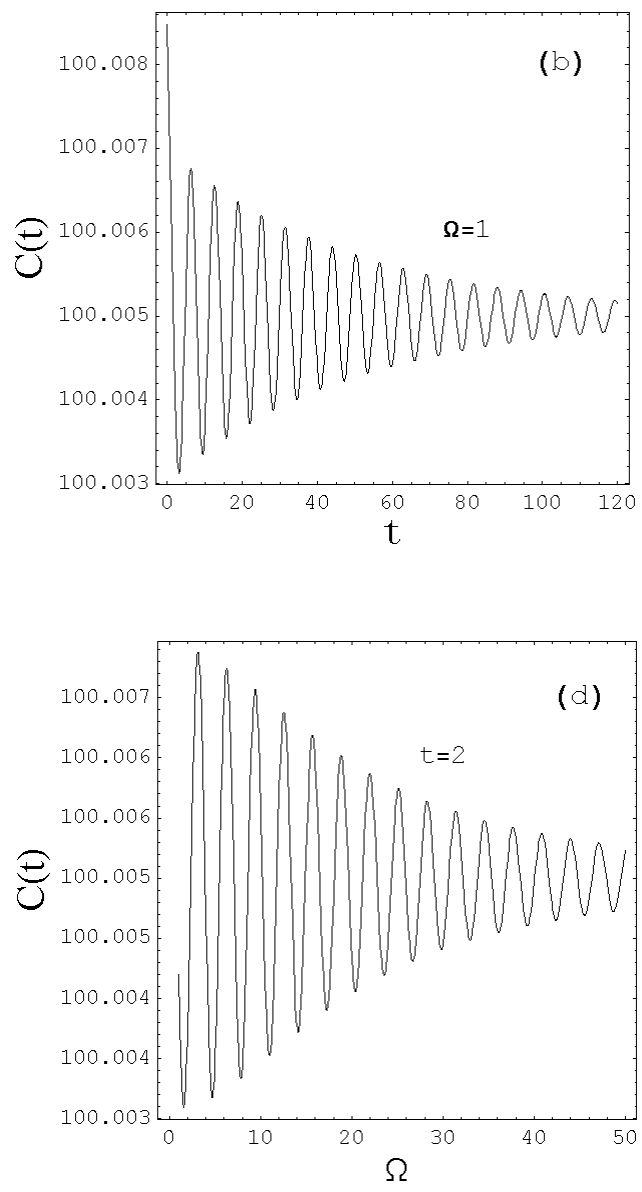

Fig.1. When $\tau>>1$, the influence of $\Omega$ on time evolution of $C(t)$. The values of the other parameter are $A=1, \quad B=20, P=0.001, Q=0.001, \quad a_{0}=10, \quad \lambda_{q}=0.5, \quad \tau=50$.

The influence of net gain $a_{0}$ When $\tau=50$, the influence of frequency $\Omega$ of modulation signal on time evolution of curve $C(t)$ can be seen in the three-dimensional plot, Fig.1(a), and in the $C(t)$ vs. $t$ curve of the sectional drawing, Figures1(b) and (c), and in the $C(t)$ vs. $\Omega$ curve of the 
sectional drawing figure 1(d). Figures 1(b) and (c) (The sectional drawing plot) show that $C(t)$ vs. $t$ curve exhibits a periodically surging with descending envelope. At a given time $t, C(t)$ vs. $\Omega$ curve also presents a periodical change with $\Omega$, as shown in Fig. 1(d). It can also be found in Figures 1 (b)-- (d) that when $\Omega$ increases, the periodical vibration frequency of $C(t)$ vs. $t$ curve becomes higher while the amplitude becomes smaller, that is, the curve descends rapidly. When the evolution time being long enough, the values of $C(t)$ descend to identical, as shown in Fig.1.

In the cases of $\tau>>1$, the time evolution processes of $C(t)$ differs greatly with one another. Since $C(t)$ has two timescales and is a periodical function of $\Omega$, the modulation signal frequency $\Omega$ and the pump noise self-correlation time $\tau$ greatly affect the time evolution of $C(t)$.

From the analysis above, we can see that when $\tau \gg 1$, whatever the value of $\Omega$ is, the time evolution process of $C(t)$ always take the form of periodically surging with descending envelope. Obviously, the modulation of colored pump noise by periodical signal is the major reason for the time evolution of intensity correlation function to have all the above-mentioned characteristics. This shows that the interaction between modulation signal and the "color" of pump noise has a great influence on the dynamic behavior of laser system.

\section{The Influence of Net Gain $a_{0}$ On the Time Evolution of $C(t)$}
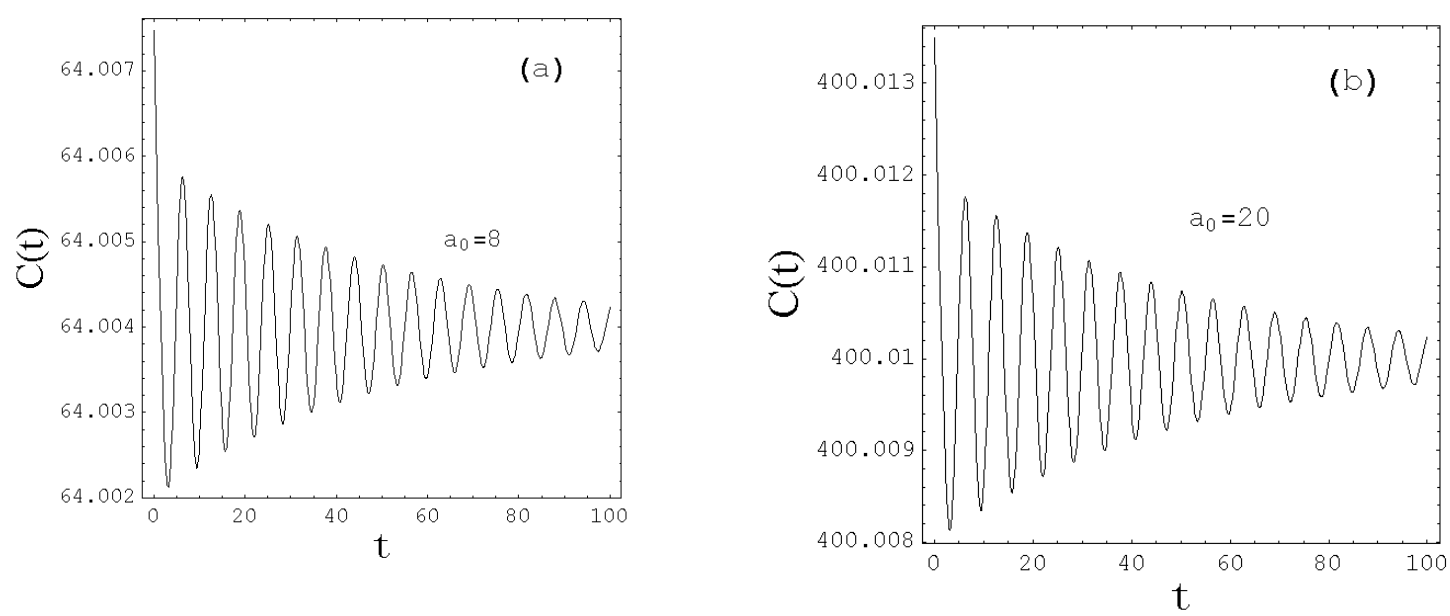

Fig.2. When $\tau \gg>1$, the influence of $a_{0}$ on time evolution of $C(t)$. The values of the other parameter are $A=1, \quad B=20, \quad P=0.001, \quad Q=0.001, \quad \lambda_{q}=0.5, \quad \tau=50, \quad \Omega=1$

Figure 2 shows, when $\tau=50$, how the net gain $a_{0}$ influences the time evolution of $C(t)$. In Fig.2, the time evolution of $C(t)$ exhibits the periodically surging with descending envelope. When $a_{0}$ increases, the amplitude and frequency of periodical vibration of $C(t)$ vs. $t$ curve does not change. However, when the evolution time is long enough, the values of $C(t)$ with different evolution processes are different as shown by Fig.2.

According to the research results of theoretical analysis, the system is simulated reasonably by Simulink software, and the theoretical research results are compared with the simulation results. It is found that the output results of the system are basically the same with the evolution process of time. The results of periodic oscillation attenuation with time

\section{Conclusion and Discussion}

To sum up , the analysis and discussion have shown: (1) In the case of $\tau>>1$, when modulation signal frequency $\Omega$ increases, the time evolution of $C(t)$ all exhibit the periodically surging with descending envelope. What's noteworthy is that when $\tau \gg 1$ (the value of $\Omega$ is unrestrained), the 
time evolution of $C(t)$ takes the form of the periodically surging with descending envelope. The physical mechanism of the phenomenon mentioned above roots in the interaction between the modulation signal and colored pump noise. This phenomenon also suggests that the modulation of the colored pump noise by the periodical signal highly affects the dynamic behavior of the laser system. (2) In the case of $\tau \gg>1$, the time evolution of $C(t)$ exhibits the periodically surging with descending envelope with the increase of $B$. (3) In the case of $\tau>>1$, when $a_{0}$ increases, the time evolution of $C(t)$ only exhibits the periodically surging with descending envelope. When the evolution time is long enough, the values of $C(t)$ corresponding to different net gain $a_{0}$ are different. This shows the great influence of the $a_{0}$ on the dynamic behavior of the laser system.

\section{Acknowledgements}

This work is supported by Science and Technology Research Project of Jiangxi Provincial Department (Grant Nos. GJJ161227、GJJ171104), the Science and Technology Research Project of Nanchang Institute of Science \& Technology (Grant Nos. NGKJ-17-05, GJKJ-16-01).

\section{References}

[1] Cheng Q H, Xu D H, Cao L, et al. (2006) Influence of net gain on the statistical fluctuation in a single-mode laser system. Chinese Optics Letters, 4 (7): 401-40.

[2] Hernandez G E, Toral R, San M M. (1990) Intensity correlation functions for the colored gain-noise model of dye lasers. Phys. Rev. A, 42(11): 6823-6830.

[3] Wang J, Zhu S Q. (1997) Effects of positive and negative coupling between noises terms in a laser-field [J]. Acta Optica Sinica, 17 (7): 884-888.

[4] Luo X Q, Zhu S Q, Gao W J. (2011) Effects of coloured noise in a two-mode laser system [J]. Chinese Physics, 10(11): 1011-1016.

[5] Cao L, Wu D J. (1994) Stochastic dynamics for systems driven by correlated noise [J]. Phys. Lett. (A), 185(1):59-64.

[6] Barzykin A V, Seki K. (1998) Periodically driven linear system with multiplicative colored noise [J]. Physical review E, 57 (6): 6555-6563. 